\title{
Review on Design Optimization of Liquid Carrier Tanker for Reduction of Sloshing Effects
}

\author{
${ }^{1}$ Sunil M Mahajan,${ }^{2}$ Ashwin D Patil,${ }^{3}$ V. N. Bartaria \\ (Asst.Professor, SITRC Sandip Foundation) \\ (PG Student of SRES-COE, Kopergaon) \\ (Professor \& HOD, Mechanical Engineering, LNCT, Bhopal)
}

\begin{abstract}
This Paper Reviews Briefly The Current Research On Sloshing And Its Effect In Liquid Carrier Tanker. The Aim Of This Paper To Study The Basics Of Sloshing And Its Prevention (Mainly In Liquid Carrier Tanker) The Liquid Sloshing Is Free Surface Fluctuation Of Liquid When Its Container Is Excited By External Vibrations Such As Earthquakes. The Liquid Sloshing May Cause Various Engineering Problem, For Example Instability Of Ships In Aero Engineering And Ocean Engineering, Failures On Structural Systems Of The Liquid Container. The Tanker Used For The Transportation Of Liquid Over The Road-Ways Is An Integral Part Of The Carrier/Vehicle. The Tanker Is Expected To Withstand The Unbalanced Forces On Account Of Transit Over Uneven And Irregular Surfaces/Contours Of The Road As Also Due To Sudden Acceleration Or Deceleration (Due To Application Of Brakes).
\end{abstract}

Keywords-Sloshing, Impact, Baffle, Simulation

\section{INTRODUCTION}

Sloshing can be defined as dynamic load acting over a tank structure as a result of the motion of a fluid with free surface confined inside the tank. Liquid sloshing is a kind of wave motion inside a partially filled tank. The sloshing phenomenon is of great practical importance to the safety of the liquid transport. Under external excitations, with large amplitude or near the natural frequency of sloshing, the liquid inside a partially filled tank is in violent oscillations. In this paper we will see the background behind the sloshing phenomena to define the problem in proper manner. Also we will see the research work carried related to this problem, proposed methodology to go for solution and scope of work.

The tanker used for the transportation of liquid over the road-ways is an integral part of the Carrier/ Vehicle. The tanker is expected to withstand the unbalanced forces on account of the transit over uneven and irregular surfaces/ contours of the road as also due to sudden acceleration or deceleration (due to application of brakes). As a result, 'sloshing' of the liquid is experienced within the tanker. Different aspects of analyses are necessary to design the tanker but sloshing analysis is also one of the prominent aspects for reducing its detrimental effects over structure of tanker. Sloshing can be the result of external forces due to acceleration/deceleration of the containment body. Of particular concern is the pressure distribution on the wall of the container reservoir and its local temporal peaks that can reach as in road tankers twice the rigid load value. In road tankers, the free liquid surface may experience large excursions for even very small motions of the container leading to stability problems. Analysis of the sloshing motion of a contained liquid is of great practical importance. Motion of a fluid can persist beyond application of a direct load to the container; the inertial load exerted by the fluid is time-dependent and can be greater than the load exerted by a solid of the same mass. This makes analysis of sloshing especially important for transportation and storage tanks. Due to its dynamic nature, sloshing can strongly affect performance and behavior of transportation vehicles, especially tankers filled with oil. In fact, a significant amount of research has gone into developing numerical models for predicting fluid behavior under various loads.

Hence liquid sloshing is a practical problem with regard to the safety of transportation systems, such as oil tankers on highways, liquid tank cars on railroads, oceangoing vessels with liquid cargo, propellant tank used in satellites and other spacecraft vehicles, and several others

\section{LITERATURE REVIEW}

Many researchers are studying the sloshing phenomena since 1950 for transportation of fluids as well as gases for better national communication. Here, in this chapter we reviewed some literature regarding sloshing and analyzing method. 
Donald $\mathrm{Liu}_{[1]}$ has reported that the normal practice is to base the tanker's design on engineering analysis, and to use rule equations only as a check, for guidance and control. The potential benefit from utilizing engineering analysis is stress verification and rational distribution of steel, which results in steel weight optimization without sacrificing strength. Also it has been suggested the different types of engineering analysis required to design the tanker which are mentioned as below:

- Ship Moment: The first step in the analysis consists of calculating the hull girder longitudinal vertical bending moment, for all anticipated loaded and ballast conditions.

- Ship Motion: The next step in the analysis is to calculate the vertical, lateral and torsional dynamic components of the hull girder bending moments, as well as the motions, point accelerations and pressure distribution along the vessel's length.

- 3-D Global 3-D Finite Element Analysis (FEM): The data obtained from ship moment and ship motion above are used to carry out a three-dimensional global FEM analysis for the entire vessel, or for a portion of the hull girder. This analysis gives the overall structural response in the form of element stresses and displacements.

- Sloshing Analysis: This analysis is used to determine the dynamic loads on the tank boundaries from the motion of the fluid within the tank due to ship movements in a seaway.

- Thermal Stress Analysis: This analysis provides the distortions and stresses in the hull structure induced by non-linear temperature differentials in vessels carrying hot cargoes.

- Fatigue and Fracture Mechanic Analysis: Based on the combined effect of loading, material properties and flaw characteristics, this analysis predict the service life of the structure, and determine the most effective inspection plan.

- Vibration Analysis: This analysis is used to determine the extent of vibrations in the ship structure induced by the interaction of fluids, structure, machinery and propellers.

Jean Ma and Mohammad Usman Jean ${ }_{[2]}$ presented that, the sloshing phenomenon in partially filled fuel tanks is more pronounced when vehicle experience a sudden start or stop. Sloshing is undesired because it produces noise, high impact force on the tank walls and the challenge of low fuel handling. Today the solution for containing sloshing is to incorporate baffles inside the tank. The presence of baffles dissipates the energy that is induced by the fuel motions. Design of baffles is necessary step during the design of a fuel tank to meet required performance specification in service.

After literature review it can be seen that, this problem is having FSI nature so the modeling and analyzing method should be susceptible to adopt such nature of problem. Here, in this chapter we will see different approaches to solve FSI problem along with explanation about FEA code useful for analysis.

\section{Approaches to solve FSI problem:}

This type of problem can be modeled in basic four approaches which are used for fluid structure interaction problem

a. Lagrangian approach

b. Euler approach

\section{Lagrangian approach}

Lagrangian formulation is usually used for describing a solid mechanics problem. The problem is described with a high number of mass particles, where the motion of every single particle is being observed in space and time. The problem is exactly defined when the motion of all the particles is known. The Lagrangian formulation is very simple and easy to use for one or only a few mass particles. However, the method becomes very complicated and complex for description of high number of mass particles. (Fig.1)

In the Eulerian formulation the problem is being observed at one point in space which does not follow the motion of the single particle. In one time step t several mass particles may pass the observed point. Their motion is exactly determined in the moment of passing through that point. In the observed point the field variables are time dependent. (Fig.2) 


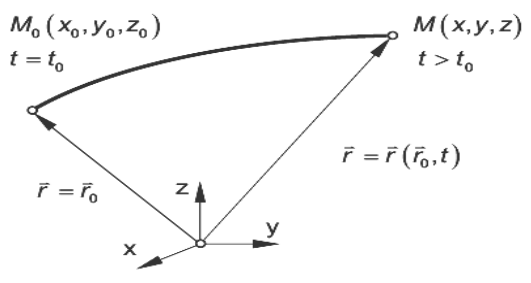

Fig. 1 Langrangian Formulation

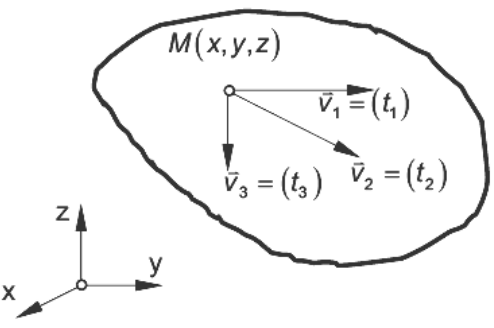

Fig. 2 Eulerian Formulation

The basic difference between the Lagrangian and the Eulerian formulation is that at the Lagrangian formulation the magnitudes $x, y$ and $z$ are variable coordinates of a moving particle. At the Eulerian formulation those coordinates represent steady coordinates of the defined field point

A Lagrangian mesh should be used to model structural parts of a problem. In the Lagrangian formulation one finite element represents the same part of the material throughout the course of the analysis. The fluid domain can be described with a material model which skips the calculation of deviatoric stresses. By defining a low bulk modulus for fluids such as water, the elastic shear forces become negligible, and by using a low yield stress, fast tran sition to plasticity can be achieved (e.g. by only considering the gravitation). Under high dynamic loading, the shear forces and any unreal introduced forces become negligible in comparison to the inertial forces of the fluid. (Fig.3) illustrates the solution process of a simple fluid problem using the Lagrangian formulation. It is presumed that the loading influences only the central node. The result of the loading is the shift of that node in a computational time step. If the influence of the loading does not stop or change, the node takes a new position in the next time step and the mesh deforms even more, since the mesh follows the material flow.

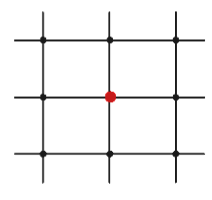

$t=0$

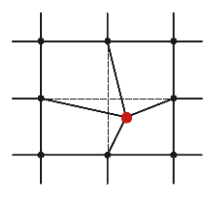

$t=\Delta t$

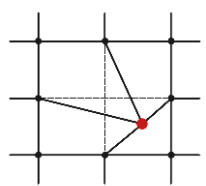

$t=2 \Delta t$

Fig. 3 Mesh Deformation in Langrangian Formulation

\section{Eulerian Approach:}

It is also possible to apply the Eulerian formulation for fluid flow analyses, where the fluid flow through the fixed mesh in a space is observed. The material point moves from one finite element to another and the finite element mesh does not move or deform. Although the Eulerian mesh in appears not to move or deform during the analysis, it does actually change its position and form only within the single time step. The reason for this is the use of Lagrangian formulation in single time steps, which is much more advanced in. The Eulerian mesh is treated in a special way (Fig.3.4). To illustrate the use of an Eulerian mesh the same example is used as in the previous chapter. Because of the central node loading, the observed node changes its position during one computational time step (mesh deforms). After the time step the analysis stops and the following two approximations are performed:

- Mesh smoothing: all the nodes of the Eulerian mesh that have been displaced due to loading are, moved to their original position;

- Advection: the internal variables (stresses, flow fields, velocity field) for all the nodes that have been moved are recomputed (interpolated) so that they have the same spatial distribution as before the mesh smoothing. In this way the mesh smoothing does not affect the internal variable distribution. 
The described procedure is being repeated for each time step of the analysis and provides the analyst with a non-movable and undeforms able Eulerian mesh.

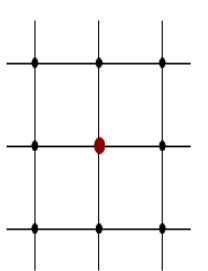

$t=0$

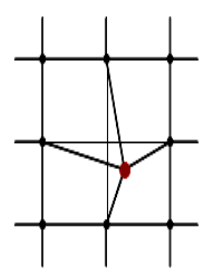

$t=\Delta t$

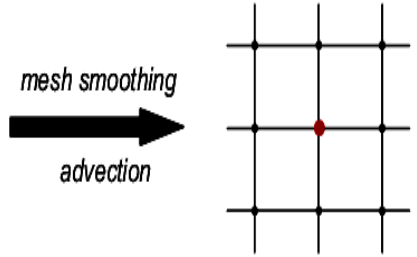

$t=\Delta t$

Fig. 4 Mesh Deformation in Eulerian Formulation

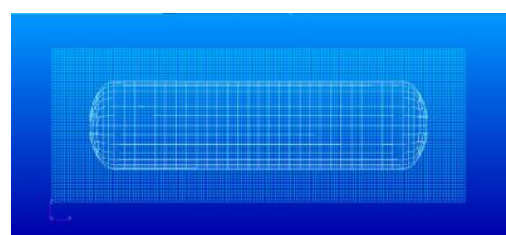

Fig.5 Aligning Euler and Langrangian Elements

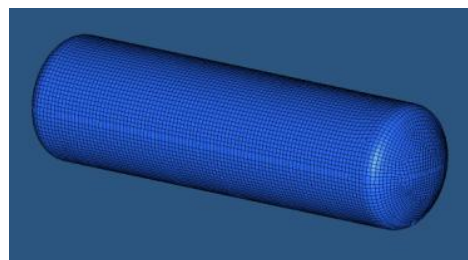

Fig.6 Tanker Mesh

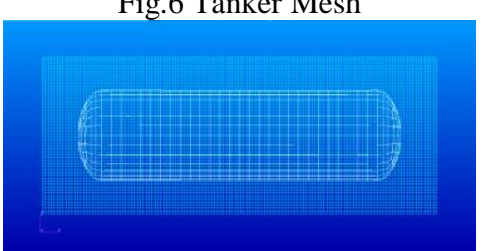

Fig.7 Meshing of LPG in Unbaffled Tank

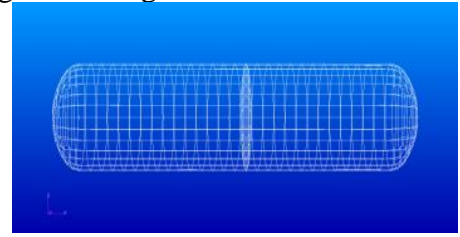

Fig.8 Meshing of LPG Tank with Full Enclosed Baffle at the

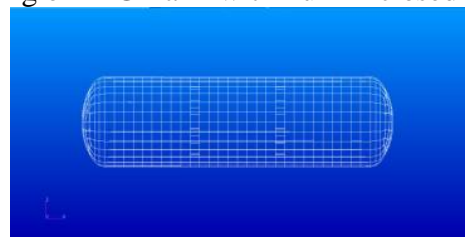

Fig.9 Meshing of LPG Tank with Two Modified Baffles

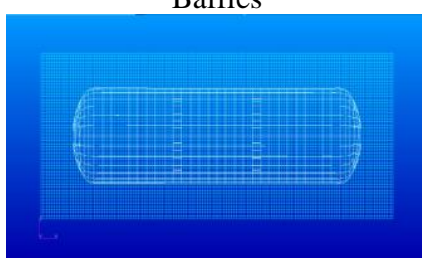

Fig.10 Eulerian and Langrangian Meshing of LPG in a Tank with Modified Baffles 


\section{Simulation of sloshing:}

Simulation results are involving different iterations in that first iteration is Sloshing of LPG without baffle.

After taking possible iterations graphs are plotted to show the pressure and velocity developed during respective condition of sloshing.

Simulation results are involving different iterations in that first iteration is Sloshing of LPG without baffle which includes various stages of sloshing simulation at start which shown in Fig.11 and Fig.12 respectively. Similarly, disturbance in fluid is clearly shown in Fig.13 and Fig.14 Fig.15 to Fig.19 shows the gradually increasing of LPG sloshing amplitude with enclosed full baffle inserted in a tanker. Similarly, Fig. 20 to Fig.24 shows the simulation of LPG sloshing with one modified baffle at the center of tank with various views. Then, simulation result of LPG sloshing with two modified baffles is given in Fig. 25 to Fig.29 with various stages of sloshing phenomena.

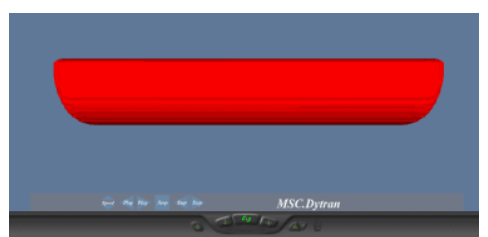

Fig.11 Iteration 1 Sloshing of LPG without Baffle

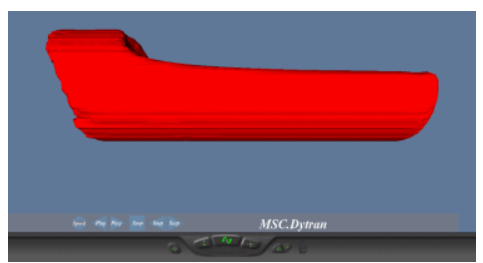

Fig.12 Iteration 1 Sloshing of LPG without Baffle in Front View

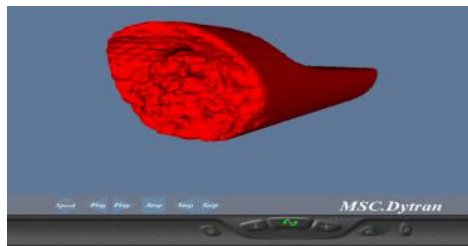

Fig.13 Iteration 1 Sloshing of LPG without Baffle showing Side View

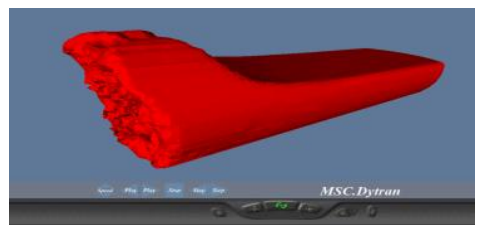

Fig.14 Iteration 1: Sloshing of LPG without Baffle showing Disturbance of Fluid

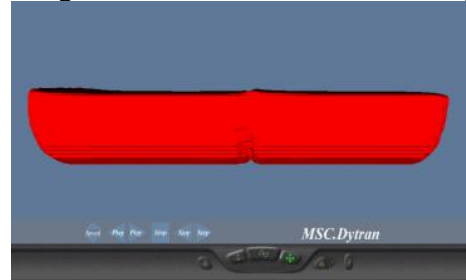

Fig.15 Iteration 2: Sloshing of LPG in a Tank with Enclosed Full Baffle

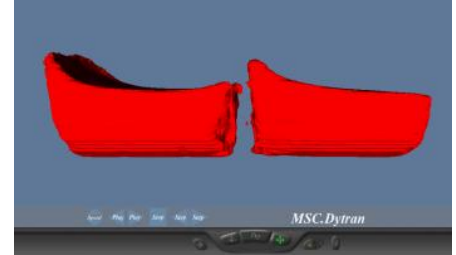

Fig.16 Iteration 2: Sloshing of LPG in a Tank with Enclosed Full Baffle at Initial Stage 


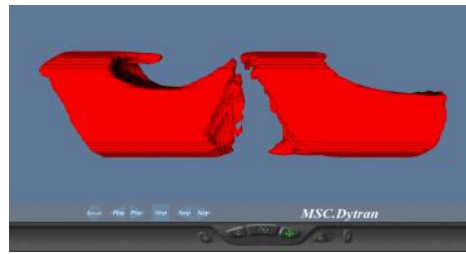

Fig.17 Iteration 2: Sloshing of LPG in a tank with enclosed full baffle at final stage

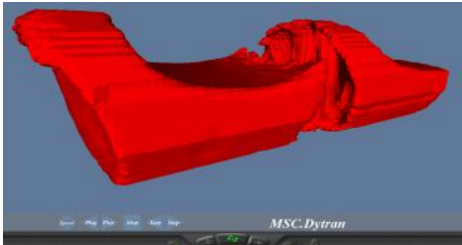

Fig.18 Iteration 2: Sloshing of LPG in a tank with enclosed full baffle showing side view

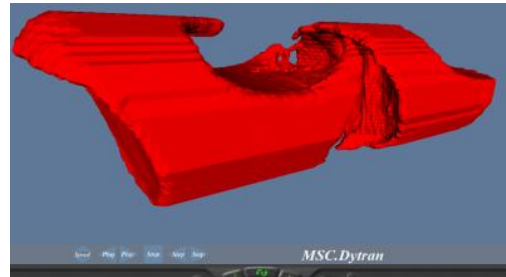

Fig.19 Iteration 2: Sloshing of LPG in a tank with enclosed full baffle showing disturbance

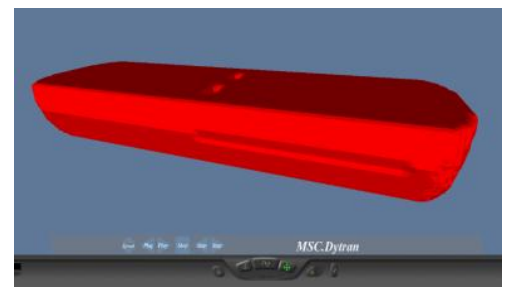

Fig.20 Iteration 3: Sloshing of LPG in a tank with one modified baffle

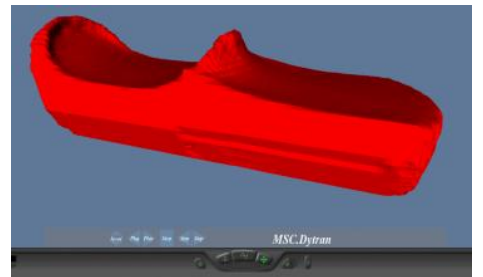

Fig.21 Iteration 3: Sloshing of LPG in a tank with one modified baffle showing lift of fluid along baffle

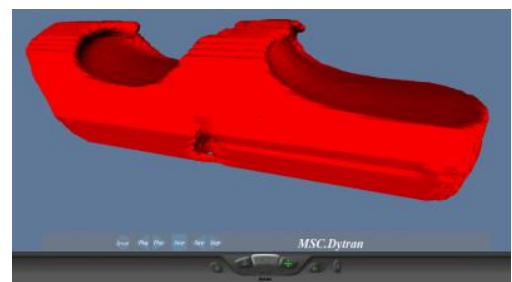

Fig.22 Iteration 3: Sloshing of LPG in a tank with one modified baffle showing peak amplitude

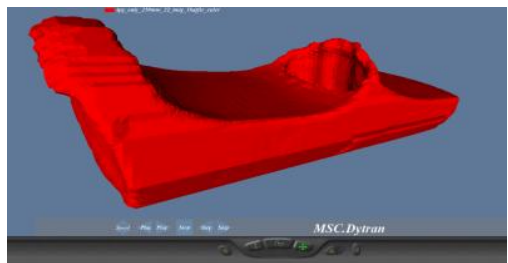

Fig.23 Iteration 3: Sloshing of LPG in a tank with one modified baffle with side view 


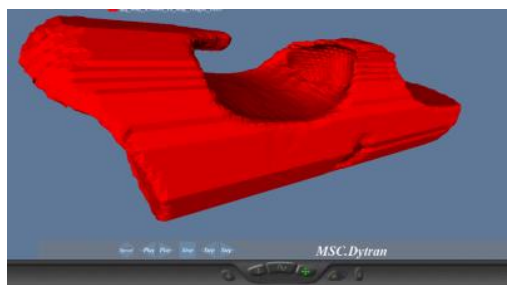

Fig.24 Iteration 3: Sloshing of LPG in a tank with one modified baffle showing disturbance of fluid in side view

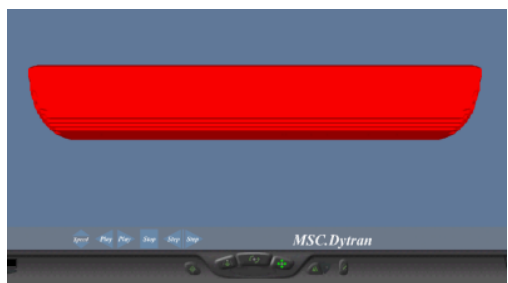

Fig.25 Iteration 4: Sloshing of LPG in a tank with two modified baffle

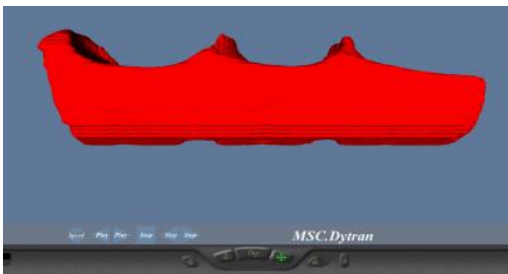

Fig.26 Iteration 4: Sloshing of LPG in a tank with two modified baffle showing lift of fluid along baffle

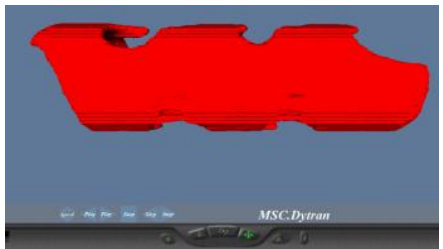

Fig.27 Iteration 4: Sloshing of LPG in a tank with two modified baffle showing peak amplitude of fluid

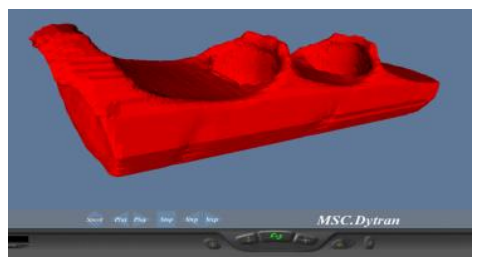

Fig.28 Iteration 4: Sloshing of LPG in a tank with two modified baffle in side view

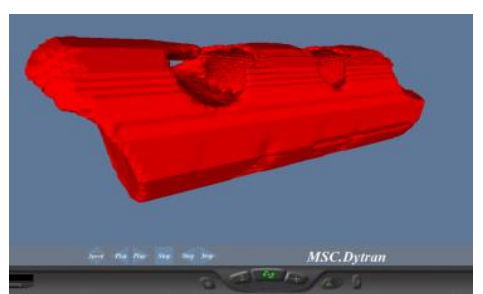

Fig.29 Iteration 4: Sloshing of LPG in a tank with two modified baffle showing disturbance of fluid

\section{Graphs:}

After taking possible iterations graphs are plotted to show the pressure and velocity developed during respective condition of sloshing 

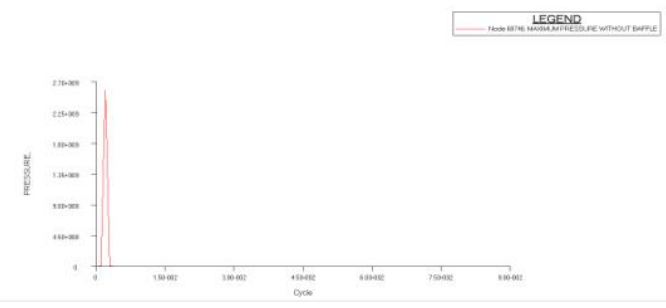

Fig.30 Sloshing of LPG without baffle
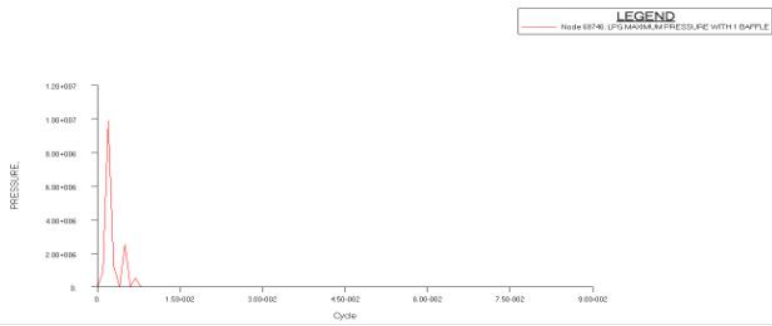

Fig.31 Sloshing of LPG in a tank with one modified baffle
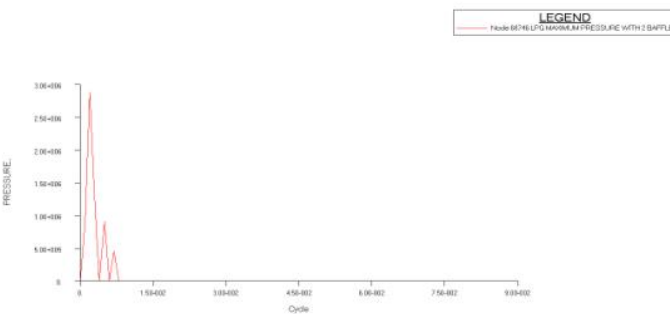

Fig.32Sloshing of LPG in a tank with two modified baffle
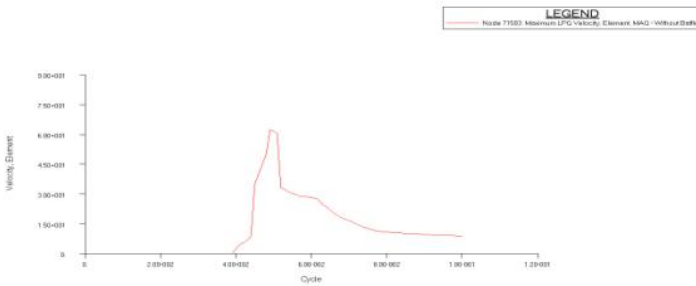

Fig.33 Sloshing of LPG without baffle

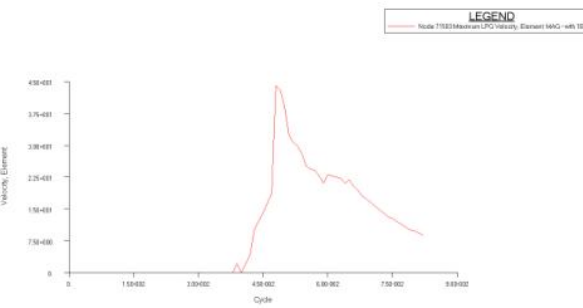

Fig.34 Sloshing of LPG in a tank with one modified baffle

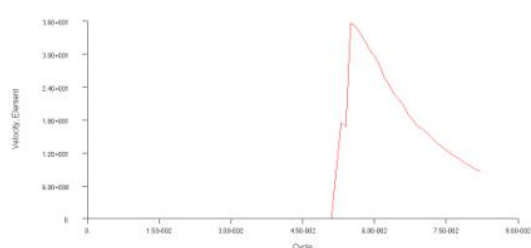

Fig.35 Sloshing of LPG in a tank with two modified baffle 


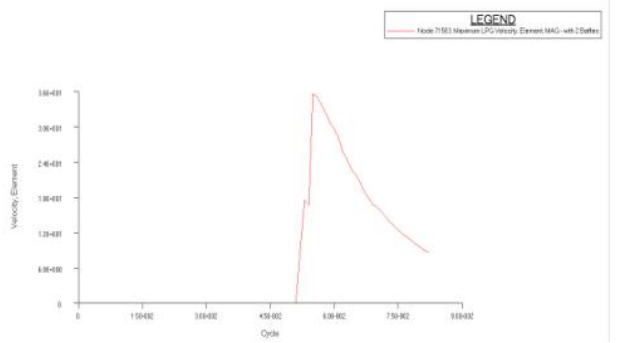

Fig.36 Sloshing of LPG in a tank with two modified baffle

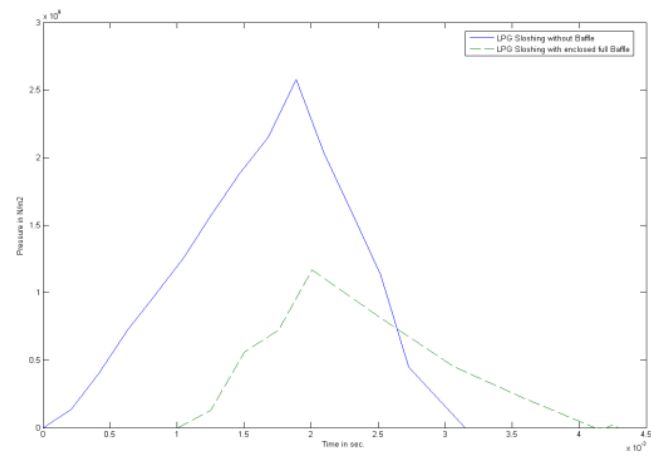

Fig.37 Comparison of LPG sloshing without baffle and with enclosed full baffle

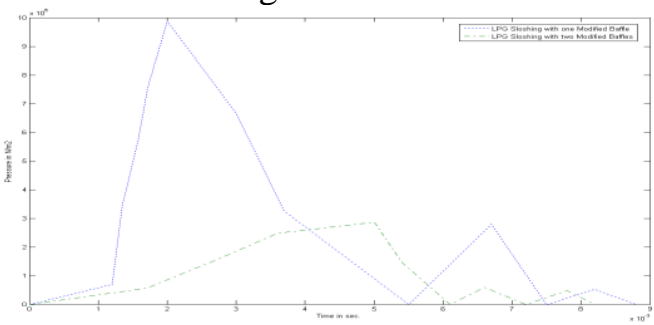

Fig.38 Comparison of LPG sloshing with one modified baffle and two modified baffles

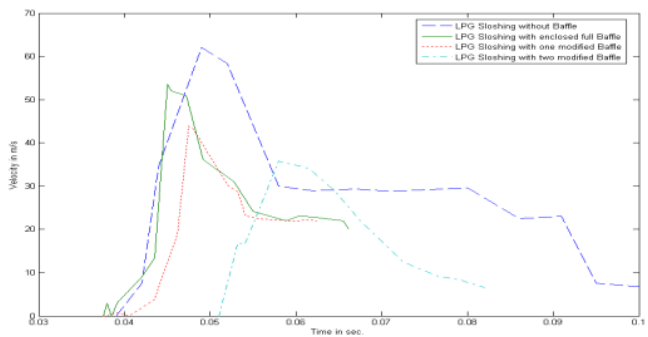

Fig.39 Comparison of velocity variations of four iterations

\section{Findings:}

From the simulation result of every iteration it can be found that, sloshing is reduced in considerable amount with the help of two modified baffles located at $2500 \mathrm{~mm}$ apart from each other. Also the maximum pressure generated in various iterations due to sloshing of fluid is as follows:

Table.1 Maximum pressure generated in various cases

\begin{tabular}{|c|c|c|c|}
\hline $\begin{array}{c}\text { Iteration } \\
\text { No. }\end{array}$ & Case Name & $\begin{array}{c}\text { Time in } \\
(\mathrm{sec})\end{array}$ & $\begin{array}{c}\text { Pressure in } \\
\left(\mathrm{N} / \mathrm{m}^{2}\right.\end{array}$ \\
\hline 1 & $\begin{array}{c}\text { LPG sloshing without } \\
\text { baffle }\end{array}$ & 0.00189 & $2.58 \times 10^{9}$ \\
\hline 2 & $\begin{array}{c}\text { LPG sloshing with enclosed } \\
\text { full baffle }\end{array}$ & 0.00200 & $1.17 \times 10^{9}$ \\
\hline 3 & $\begin{array}{c}\text { LPG sloshing with one } \\
\text { modified baffle }\end{array}$ & 0.0020 & $9.88 \times 10^{6}$ \\
\hline 4 & $\begin{array}{c}\text { LPG sloshing with two } \\
\text { modified baffles }\end{array}$ & 0.0050 & $2.87 \times 10^{6}$ \\
\hline
\end{tabular}


Similarly the maximum velocity developed in various iterations because of sloshing of fluid is as follows:

Table.2 Maximum velocity developed in various cases

\begin{tabular}{|c|l|c|c|}
\hline $\begin{array}{c}\text { Iteration } \\
\text { No. }\end{array}$ & \multicolumn{1}{|c|}{ Case Name } & $\begin{array}{c}\text { Time in } \\
\text { sec. }\end{array}$ & $\begin{array}{c}\text { Velocity in } \\
\mathrm{m} / \mathrm{s}\end{array}$ \\
\hline 1 & $\begin{array}{l}\text { LPG sloshing } \\
\text { without baffle }\end{array}$ & 0.049 & 62.13 \\
\hline 2 & $\begin{array}{l}\text { LPG sloshing with } \\
\text { enclosed } \\
\text { full baffle }\end{array}$ & 0.045 & 53.48 \\
\hline 3 & $\begin{array}{l}\text { LPG sloshing with } \\
\text { one } \\
\text { modified baffle }\end{array}$ & 0.0475 & 43.85 \\
\hline 4 & $\begin{array}{l}\text { LPG sloshing with } \\
\text { two } \\
\text { modified baffles }\end{array}$ & 0.058 & 35.8 \\
\hline
\end{tabular}

Therefore, from Table 1 and 2 we can say that, more time is required to generate maximum pressure and velocity in the case of two modified baffles compared to other cases. Also the maximum stress developed in modified baffle having $20 \mathrm{~mm}$ thickness is $2.20 \times 10^{8} \mathrm{~Pa}$ which is lower than the yield strength of baffle material (ASTM A576). Similarly, stress on wall of tanker is $2.05 \times 10^{8} \mathrm{~Pa}$ lower than yield strength of tanker material (AISI 1040).

\section{CONCLUSION}

Analysis of cylindrical liquid carrier tanker is carried out using the finite element method. Studies of various methods in FEA are done and one particular method is selected to model fluid-structure interaction problem. These interaction problems are quite complex and they have been challenging as well.

Using MSc-Dytran software defined problem has been modelled which uses Arbitrary Langrangian Eulerian method (ALE). Elements depicting the properties of the tanker and the liquid are selected and coupled. Water is selected first to see the nature of sloshing and to get the maximum pressure. Then iterations are taken for real liquid in the problem i.e. Liquefied Petroleum Gas (LPG). Analysis is done to obtain sloshing patterns, pressure and velocity parameters in different cases. From the results that obtained in analysis following conclusions can be drawn

We can accept the challenge for transportation of liquid in partially filled tankers by using baffles in proper shape, numbers and location. In this problem sloshing of LPG is reduced in half filled cylindrical tanker at the speed of $40 \mathrm{kmph}$ by using two modified baffles. Also effect of sloshing over tanker and baffles are decreased with proper thickness. The pressure and velocity developed in two baffled condition is lower than unbaffled, one baffled and enclosed baffled condition. So it is recommended to use two modified baffles, $2500 \mathrm{~mm}$ apart from each other with thickness $20 \mathrm{~mm}$ which can decrease the sloshing considerably and sustain the sloshing pressure

\section{REFERENCES}

[1]. Donald Liu "Tanker Spills Prevention By Design" National academic Press, Publication Year1991 ISBN-10: 0-309-04377-8 ,pp.208 to 213

[2]. Jean Ma and Mohammad Usman Jean "Modeling of Fuel Sloshing Phenomenon Considering Solid-Fluid Interaction" $8^{\text {TH }}$ International LSDYNA Users Conference Fluid/Structure

[3]. Ranjit Babar and V.Katkar "Simulation of Fuel Tank Slosh Test-Coupled Eulerian-Langrangian Approach" Tata Technologies, TATA motors Ltd. Pimpri, Pune

[4]. Kim Hyun-Soo and Lee Young-Shin "Optimization design technique for reduction of sloshing by evolutionary methods" Journal of Mechanical Science and Technology,Number1/January 2008,Vol.22

[5]. Jang-Ryong Shin and Kyungsik Choi and Sin-Young Kang "An Analytical Solution to Sloshing Natural Periods for a Prismatic Liquid Cargo Tank with Baffles" Proceeding of Sixteenth International Offshore and Polar Engineering Conference, California, USA, May 28June 2, 2006 